\title{
Variable stars in the Galactic center, as revealed by the VVV Survey
}

\author{
Claudio Navarro Molina ${ }^{1,2, \star}$, Jura Borissova ${ }^{1,2}$, Márcio Catelan ${ }^{3,2}$, Radostin Kurtev $^{1,2}$, and \\ Nicolás Medina ${ }^{2}$ \\ ${ }^{1}$ Universidad de Valparaíso, Instituto de Física y Astronomía, Av. Gran Bretaña 1111, Playa Ancha, Casilla \\ 5030, Valparaíso, Chile \\ ${ }^{2}$ Millennium Institute of Astrophysics, Santiago, Chile \\ ${ }^{3}$ Pontificia Universidad Católica de Chile, Instituto de Astrofísica, Av. Vicuña Mackenna 4860, 782-0436 \\ Macul, Santiago, Chile
}

\begin{abstract}
A variability search has been performed in the Galactic center, using the nearinfrared images from the Vista Variables in the Vía Láctea (VVV) Survey. Light curves contain 89 epochs in the $K_{S}$ band. A total of 353 variable stars were found, of which only 47 are already present in the literature.
\end{abstract}

\section{Introduction}

The Galactic center (GC) is an exceptional laboratory to test the formation and evolution of stars under extreme conditions. Three massive and young star clusters have been found in this region: the Arches, Quintuplet, and the Nuclear Cluster surrounding Sagittarius A*. The Quintuplet cluster is located at a projected distance of about $30 \mathrm{pc}$ from Sgr A*. It has an age of $3.5 \mathrm{Myr}$ and a mass of $\sim 10^{4} \mathrm{M}_{\odot}$. On the other hand, at only 5 arcmin from Quintuplet, we find Arches. This is the youngest of the three central clusters, with an age of $\sim 2.5 \mathrm{Myr}$.

In order to study the stellar population of this region, we need near-infrared observations, due to the extremely high visual extinction $\left(A_{V} \sim 30\right.$ magnitudes) along the line-of-sight. To achieve this goal, we have used the VVV ESO Public Survey ([4]).

Here we present our first results of the variability search around Arches and Quintuplet.

\section{Methodology and results}

VVV images are obtained with the 4.1-m VISTA telescope at Cerro Paranal, Chile. The total survey area of $562 \mathrm{deg}^{2}$ is divided into 348 tiles. For this work, we used tile b333, located at the GC, in particular in a region covered by a single chip, which is $11.5^{\prime} \times 11.5^{\prime}$ in size. Images were automatically reduced by the Cambridge Astronomical Survey Unit (CASU) pipeline ([2]). A total of 84 epochs of $K_{S}$ images were used, corresponding to observations between 2010 and 2015.

\footnotetext{
${ }^{\star}$ claudio.navarro@postgrado.uv.cl
} 

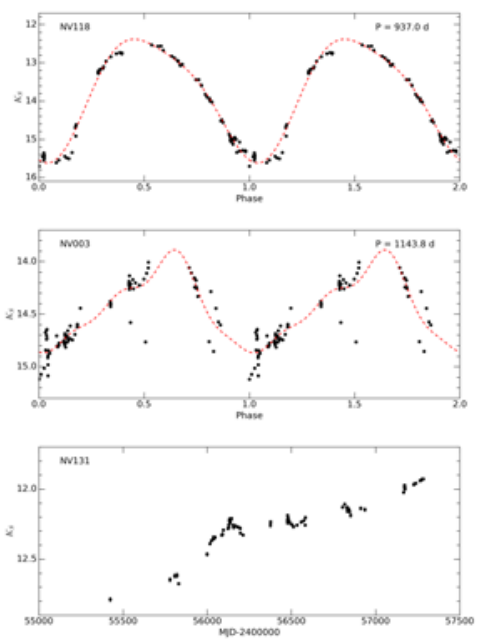

Figure 1. Typical light curves found in this work. Top panel shows a Mira light curve, with a period of 937 days. Middle panel displays a semi-regular variable with a primary period of 1143.8 days. Finally, the bottom panel shows an eruptive variable star candidate, possibly of EXor type.

In order to deal with the extreme crowding present in the images, we performed point-spread function (PSF) fitting photometry of the images. For this, we used DoPHOT ([1]). Further details about the methodology can be found in [5].

After a master catalogue was built from the 89000 sources found on the images, we performed a variability analysis using the Stetson J index ([6]), adapted to single-band time series. 654 candidates were found with this method. A visual inspection of all candidates was performed in order to remove false positives due to blending or image artifacts. Finally, we obtained 353 variable stars. In order to estimate a time interval for their variations, a generalized Lomb-Scargle periodogram was used.

Our 353 variable stars have been cross-matched with the data found in the literature, namely the Mira search carried out by [3]. 47 counterparts were found, meaning that we have discovered 306 new variables in the region. Most of the variable stars correspond to asymptotic giant branch (AGB) stars, like Miras, semi-regulars, and irregulars; in addition, some eruptive variables have been found. Examples of light curves are shown in Figure 1. From the first 50 variables that were analyzed, 4 correspond to Miras, 21 to semi-regulars, while the remaining 25 have irregular light curves. The crossmatch with [3] allowed us to have a long time baseline (over 12 years), enough to check for secular variations in their light curves, which are expected for stars in the AGB phase, both in their amplitude and/or periodicity.

\section{References}

[1] Alonso-García, J., Mateo, M., Sen, B., Banerjee, M., Catelan, M., Minniti, D., \& von Braun, K., AJ, 143, 70 (2012)

[2] Irwin, M. J., Lewis, J., Hodgkin, S., et al., in Optimizing Scientific Return for Astronomy through Information Technologies, ed. P. J. Quinn \& A. Bridger, Procceedings of the SPIE, 5493, 411 (2004)

[3] Matsunaga, N., Kawadu, T., Nishiyama, S., Nagayama, T., Hatano, H., Tamura, M., Glass, I. S., \& Nagata, T., MNRAS, 399, 1709 (2009)

[4] Minniti, D., Lucas, P. W., Emerson, J. P., et al., New Astron., 15, 433 (2010)

[5] Navarro Molina, C., Borissova, J., Catelan, M., et al., MNRAS, 462, 1180 (2016)

[6] Stetson, P. B., PASP, 108, 851 (1996) 